\title{
A 3-D current system of an ionospheric dynamo including a skin effect in an anisotropic plasma
}

\author{
V. V. Plotkin \\ Institute of geophysics SB RAS, 630090, Novosibirsk, Koptuyg av., 3, Russia \\ (Received March 17, 2004; Revised September 27, 2004; Accepted December 15, 2004)
}

\begin{abstract}
The numerical two-mode electromagnetic model of an ionospheric dynamo is constructed taking into account a skin effect of a field in an anisotropic plasma, i.e. a non-potential electric field of Sq-variations in the ionosphere. It is shown that a three-dimensional current system generated by the dynamo action of winds is located at altitudes less than about $200 \mathrm{~km}$ basically. It does not depend on an ionosphere condition in upper layers and weaker field-aligned currents between the northern and southern hemispheres practically, and an almost complete closed current circuit is generated in the dynamo layer.
\end{abstract}

Key words: Ionospheric dynamo, skin effect, non-potential electric field, Sq-variation.

\section{Introduction}

Recent models of ionospheric current systems generated by the dynamo action of winds have improved in achieving more realistic solutions progressively. Richmond and Roble (1987) used the Thermospheric General Circulation Model (NCAR/TGCM) with semidiurnal tidal forcing at the lower boundary. Richmond et al. (1992) used a new simulation model of upper atmospheric dynamics (NCAR/TIE-GCM), taking into account the self-consistent electrodynamic interactions between the thermosphere and ionosphere. The every possible simplifications and approximations used at different stages of ionospheric dynamo studies are specified, for example, by Singh and Cole (1987). A necessity of three-dimensional efficient numerical model allowing to carry out exact, stable and economic computations also is marked. It is specified, that the first steps in this direction are made by Takeda and Maeda (1980) and Takeda (1982).

These models are based on the assumption of infinite longitudinal conductivity and equipotential geomagnetic field lines in the ionosphere. It allowed an integration along geomagnetic field lines of the initial equation for a total current divergence to receive the 2-D equation for a potential. The field-aligned currents at the upper boundary of the ionosphere are zero from the assumption of symmetry between northern and southern hemispheres. If the symmetry was absent, it is assumed that these currents flow entirely into the ionosphere of the opposite hemisphere at the conjugate points.

Let us note here two circumstances. First, generally speaking, it is necessary to take into account a skin effect at the large values of the conductivity in the ionosphere. Secondly, the assumption that the field-aligned currents reach as a whole the ionosphere of the opposite hemisphere is rep-

Copy right(c) The Society of Geomagnetism and Earth, Planetary and Space Sciences (SGEPSS); The Seismological Society of Japan; The Volcanological Society of Japan; The Geodetic Society of Japan; The Japanese Society for Planetary Sciences; TERRAPUB. resented not so clear. For example, a determination of fieldaligned currents causes difficulties. Richmond and Roble (1987) have noted that their pattern and the patterns of fieldaligned currents by Takeda (1982) and Stening (1977) differ greatly from each other and that the computation of fieldaligned currents is quite sensitive to details of a numerical model. There is a question, whether a certain potential in one hemisphere can affect the conjugated point of other hemisphere through a geomagnetic force line. In the present paper, it is supposed to analyse, as far as essential is this galvanic connection between northern and southern hemispheres for the ionospheric dynamo assuming a skin attenuation of fields. With this purpose, it is supposed to refuse an approximation of equipotential geomagnetic field lines and a potential electrical field in general, taking into account a skin effect in the ionosphere, as well as in the Earth (e.g. Rokityansky, 1982). Assuming further to use the model of an ionospheric source for the magnetovariation and magnetotelluric sounding the non-uniform Earth, a global problem of an ionospheric dynamo was considered in the present paper including all layers namely the ionosphere, the atmosphere and the Earth.

A mode approach for an electromagnetic field is used in this global task allowing to find the numerical solution at an arbitrary distribution of external currents and conductivity heterogeneities of a medium. The connection between magnetic and electric modes of a field is taken into account in the anisotropic ionosphere with a dependence of medium parameters on angular spherical coordinates.

\section{The Description of a Method and Basic Param- eters of a Model}

Investigating a global electromagnetic field, it is convenient to present the field as the sum of electric and magnetic modes. These modes appear independent in a spherical symmetric and isotropic medium (e.g. Rokityansky, 1982). Disturbances of a spherical symmetry and anisotropic conductivities result in necessity to take into account intercon- 

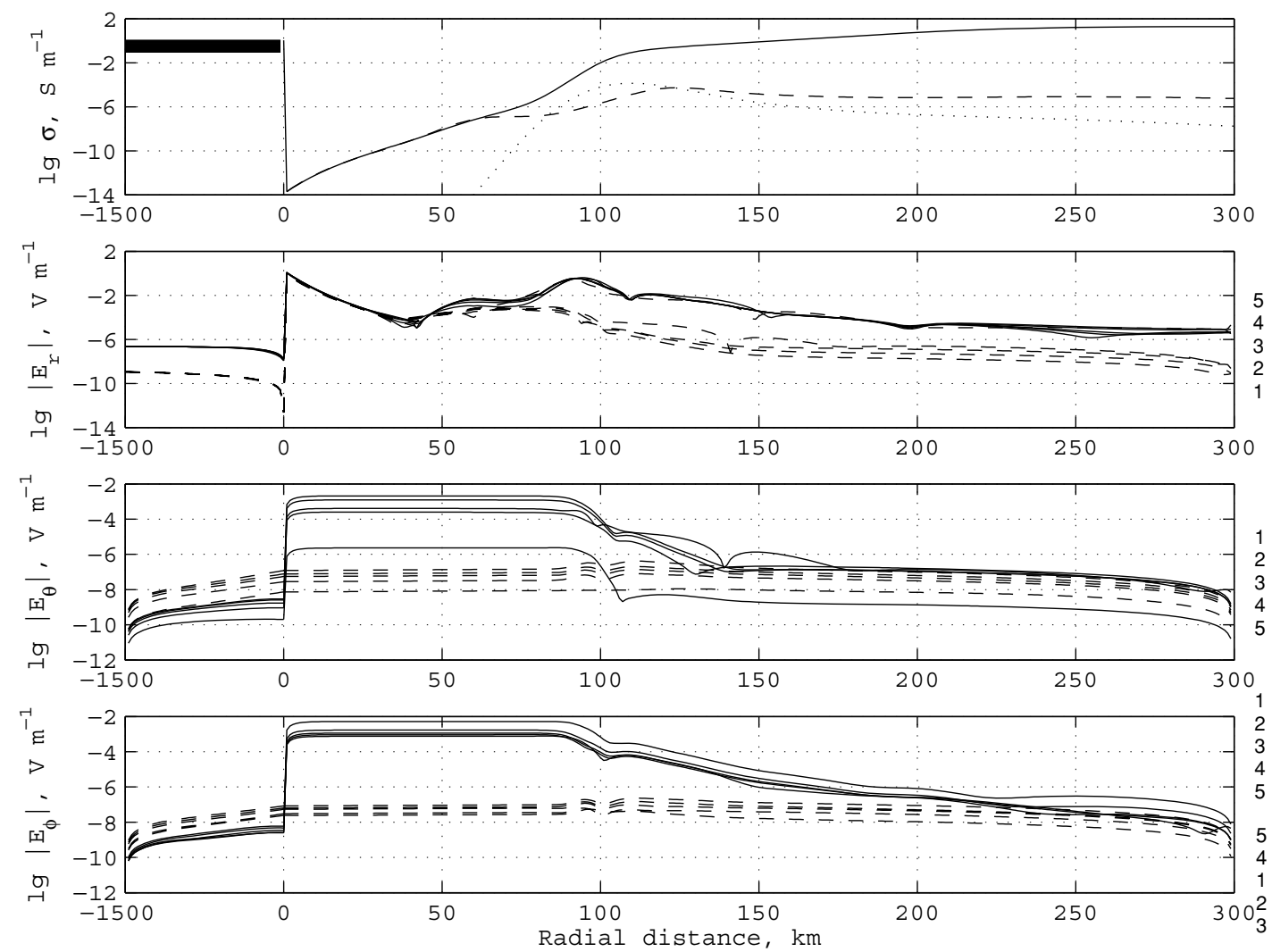

Fig. 1. Radial profiles of longitudinal (solid line), Pedersen (dashed line) and Hall (dotted line) conductivities used at simulations, and calculated high-altitude profiles of component moduli of the electric field of both modes generated in medium by the dynamo action of winds for longitude $0^{\circ}$ and several latitudes (the arrangement of lines for latitudes are specified by numbers, which correspond 1 to $80^{\circ} \mathrm{N}, 2$ to $60^{\circ} \mathrm{N}, 3$ to $40^{\circ} \mathrm{N}, 4$ to $20^{\circ} \mathrm{N}$ and 5 to $0^{\circ}$, solid lines show potential parts of the electric field, dashed lines show vortical parts of horizontal electric field components and the radial electric field component including its vortical part). Radial distances from the Earth's surface are indicated.

nections of electric and magnetic modes. To receive equations describing these interconnections of modes, it is possible to take advantage of the following performance for horizontal components of electrical $\mathbf{E}(\mathbf{r}, t)$ and magnetic $\mathbf{H}(\mathbf{r}, t)$ fields:

$$
\begin{aligned}
& E_{\theta}=\frac{1}{r} \frac{\partial E^{(1)}}{\partial \theta}+\frac{1}{r \sin \theta} \frac{\partial E^{(0)}}{\partial \varphi}, \\
& E_{\varphi}=\frac{1}{r \sin \theta} \frac{\partial E^{(1)}}{\partial \varphi}-\frac{1}{r} \frac{\partial E^{(0)}}{\partial \theta}, \\
& H_{\theta}=\frac{1}{r} \frac{\partial H^{(1)}}{\partial \theta}+\frac{1}{r \sin \theta} \frac{\partial H^{(0)}}{\partial \varphi}, \\
& H_{\varphi}=\frac{1}{r \sin \theta} \frac{\partial H^{(1)}}{\partial \varphi}-\frac{1}{r} \frac{\partial H^{(0)}}{\partial \theta} .
\end{aligned}
$$

Here $r, \theta$ and $\varphi$ are spherical coordinates of a point in system with the origin at the centre of the Earth, $E^{(1)}$ and $H^{(0)}$ are scalar potentials of electrical and magnetic fields of an electric mode, $E^{(0)}$ and $H^{(1)}$ are similar potentials of electrical and magnetic fields of a magnetic mode. Let us substitute (1) in the Maxwell's initial equations:

$$
\nabla \times \mathbf{H}=\mathbf{j}+\mathbf{J}, \quad \nabla \times \mathbf{E}=-\mu_{0} \frac{\partial \mathbf{H}}{\partial t},
$$

where $\mu_{0}$ is the magnetic permeability, $\mathbf{J}(\mathbf{r}, t)$ is the density of an external current, also it is supposed that $\mathbf{j}=\widehat{\sigma} \mathbf{E}$ is the density of a current in a conducting medium, where $\widehat{\sigma}(\mathbf{r})$ is the tensor of the conductivity. The currents of displacement are not taken into account in the first equation of system (2), as here we shall be limited to consideration of low-frequency fields basically of diurnal variations of fields. The radial components of the equations (2) give:

$$
-\frac{1}{r^{2}} \Delta_{\Omega} H^{(0)}=j_{r}+J_{r}, \quad \frac{1}{r^{2}} \Delta_{\Omega} E^{(0)}=\mu_{0} \frac{\partial H_{r}}{\partial t} .
$$

Here

$$
\Delta_{\Omega}=\frac{1}{\sin \theta} \frac{\partial}{\partial \theta} \sin \theta \frac{\partial}{\partial \theta}+\frac{1}{\sin ^{2} \theta} \frac{\partial^{2}}{\partial \varphi^{2}}
$$

is the Laplacian operator angular part. The horizontal components of the equations (2) lead to additional connections between potentials of magnetic and electrical fields of modes:

$$
\frac{\partial E^{(0)}}{\partial r}=-\mu_{0} \frac{\partial H^{(1)}}{\partial t}, \quad E_{r}-\frac{\partial E^{(1)}}{\partial r}=-\mu_{0} \frac{\partial H^{(0)}}{\partial t} .
$$

Using relations (1)-(4) it is possible to receive equations describing fields of electrical and magnetic modes:

$$
\begin{aligned}
& \frac{1}{r^{2}} \Delta_{\Omega}\left(\frac{\partial^{2} E^{(0)}}{\partial r^{2}}+\frac{1}{r^{2}} \Delta_{\Omega} E^{(0)}\right) \\
& +\mu_{0} \frac{\partial}{\partial t}(\nabla \times(\mathbf{j}+\mathbf{J}))_{r}=0
\end{aligned}
$$



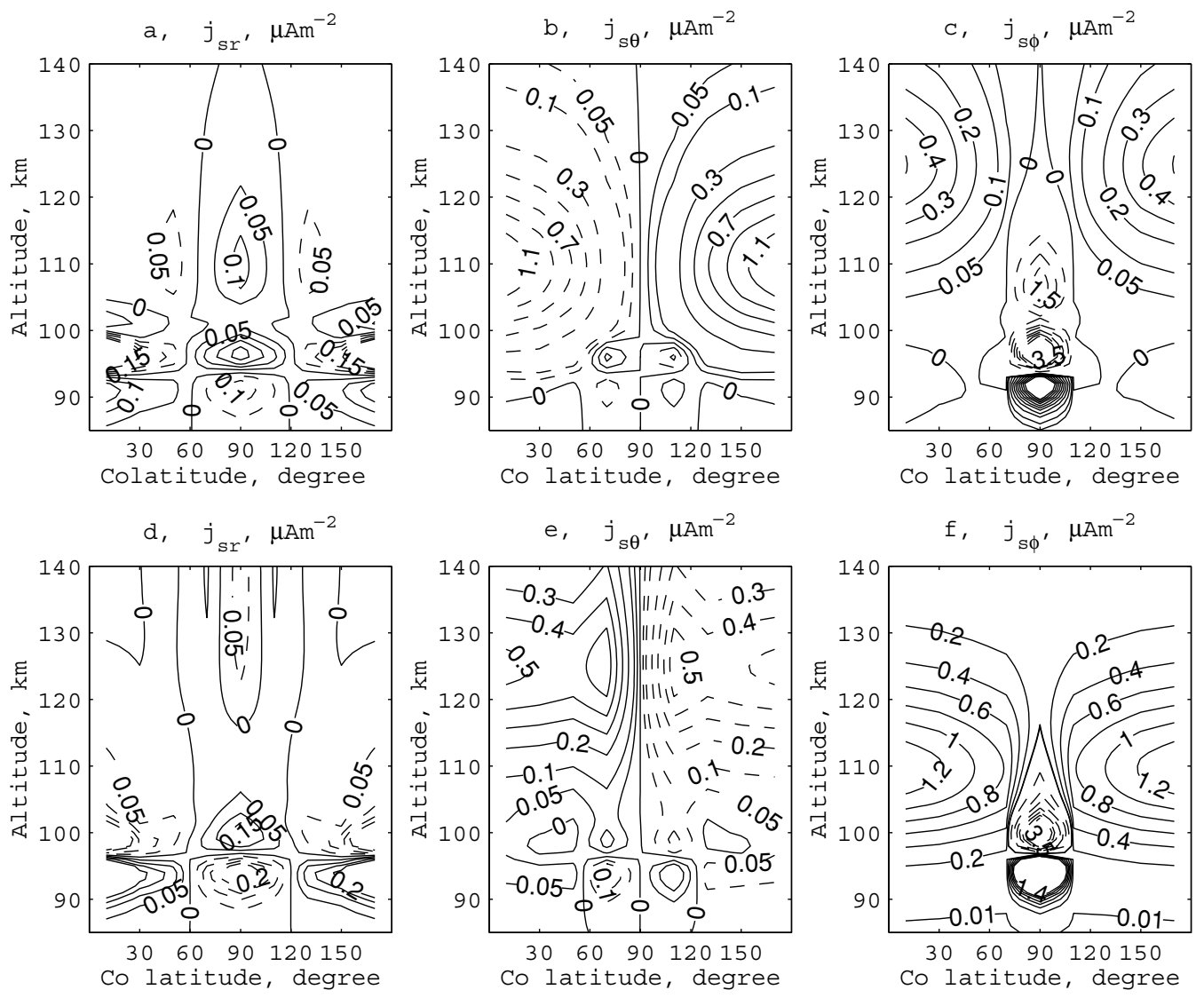

Fig. 2. Total current distributions of the ionospheric dynamo in view of a vortical electric field for midnight - midday (a, b and c) and morning - evening (d, e and f) meridians. Contours of moduli of components of total ionospheric current are shown for $j_{s r}$ component (a and d), for $j_{s} \theta$ component (b and e) and for $j_{s \varphi}$ component (c and f). Solid and dashed lines correspond to positive and negative values respectively for midnight and morning meridians, for midday and evening meridians the component signs vary on opposite.

$$
\begin{gathered}
\nabla \cdot(\mathbf{j}+\mathbf{J})=0 \\
\frac{1}{r^{2}} \Delta_{\Omega}\left(E_{r}-\frac{\partial E^{(1)}}{\partial r}\right)=\mu_{0} \frac{\partial}{\partial t}\left(j_{r}+J_{r}\right), \\
j_{r}=\sigma_{r r} E_{r}+\sigma_{r \theta} E_{\theta}+\sigma_{r \varphi} E_{\varphi}, \\
j_{\theta}=\sigma_{\theta r} E_{r}+\sigma_{\theta \theta} E_{\theta}+\sigma_{\theta \varphi} E_{\varphi}, \\
j_{\varphi}=\sigma_{\varphi r} E_{\varphi}+\sigma_{\varphi \theta} E_{\theta}+\sigma_{\varphi \varphi} E_{\varphi} . \\
\sigma_{r r}=\sigma_{0} \cos ^{2} \chi+\sigma_{P} \sin ^{2} \chi \\
\sigma_{r \theta}=\left(\sigma_{0}-\sigma_{P}\right) \cos \chi \sin \chi \cos D-\sigma_{H} \sin \chi \sin D, \\
\sigma_{r \varphi}=\left(\sigma_{0}-\sigma_{P}\right) \cos \chi \sin \chi \sin D+\sigma_{H} \sin \chi \cos D, \\
\sigma_{\theta r}=\left(\sigma_{0}-\sigma_{P}\right) \cos \chi \sin \chi \cos D+\sigma_{H} \sin \chi \sin D, \\
\sigma_{\theta \theta}=\left(\sigma_{0}-\sigma_{P}\right) \sin { }^{2} \chi \cos { }^{2} D+\sigma_{P}, \\
\sigma_{\theta \varphi}=\left(\sigma_{0}-\sigma_{P}\right) \sin ^{2} \chi \sin D \cos D-\sigma_{H} \cos \chi, \\
\sigma_{\varphi r}=\left(\sigma_{0}-\sigma_{P}\right) \cos \chi \sin \chi \sin D-\sigma_{H} \sin \chi \cos D, \\
\sigma_{\varphi \theta}=\left(\sigma_{0}-\sigma_{P}\right) \sin ^{2} \chi \sin D \cos D+\sigma_{H} \cos \chi \\
\sigma_{\varphi \varphi}=\left(\sigma_{0}-\sigma_{P}\right) \sin ^{2} \chi \sin ^{2} D+\sigma_{P} .
\end{gathered}
$$

(6) zenith angle $\chi$ in spherical system of coordinates (Plotkin, 2003). The equation (5) determines a magnetic mode po(7) tential $E^{(0)}$. Electrical mode fields satisfy the equations (6) and (7). These modes are independent in a spherically symmetric isotropic medium. They are generated to vortical $(\nabla \times \mathbf{J} \neq 0)$ and irrotational $(\nabla \cdot \mathbf{J} \neq 0)$ parts of external currents, respectively. As it is visible from equation (7), the electrical mode is generated also by a radial external current. The specified modes are connected with each other at presence of the anisotropy and angular dependence of the conductivity on sphere. Each of modes generates other mode according to the equations (5)-(8). It is possible to note, that the equation of the fourth order (5) for a field of a magnetic mode is reduced to the Helmholtz equation only at the weak dependence of conductivity on angles (when derivatives are small appropriate). As it is visible from equations (1) and (7), the electric field of a magnetic mode is vortical, and the electric field of an electrical mode is potential and

$$
E_{r}=\frac{\partial E^{(1)}}{\partial r}
$$

The conductivity becomes anisotropic in the upper layers of an atmosphere and in the ionosphere. The conductivity tensor components depend on values of longitudinal $\sigma_{0}$, Pedersen $\sigma_{P}$ and Hall $\sigma_{H}$ conductivities of a plasma in a geomagnetic field with the local declination $D$ and the only for low frequencies $\omega$ when a condition $\mu_{0} \omega \sigma L^{2} \ll 1$ is valid, where $L$ is a characteristic scale.

The numerical solution of system of equations (5)-(8) was carried out by using the matrix flow run technique along the radial coordinate. Introduction of flows according 

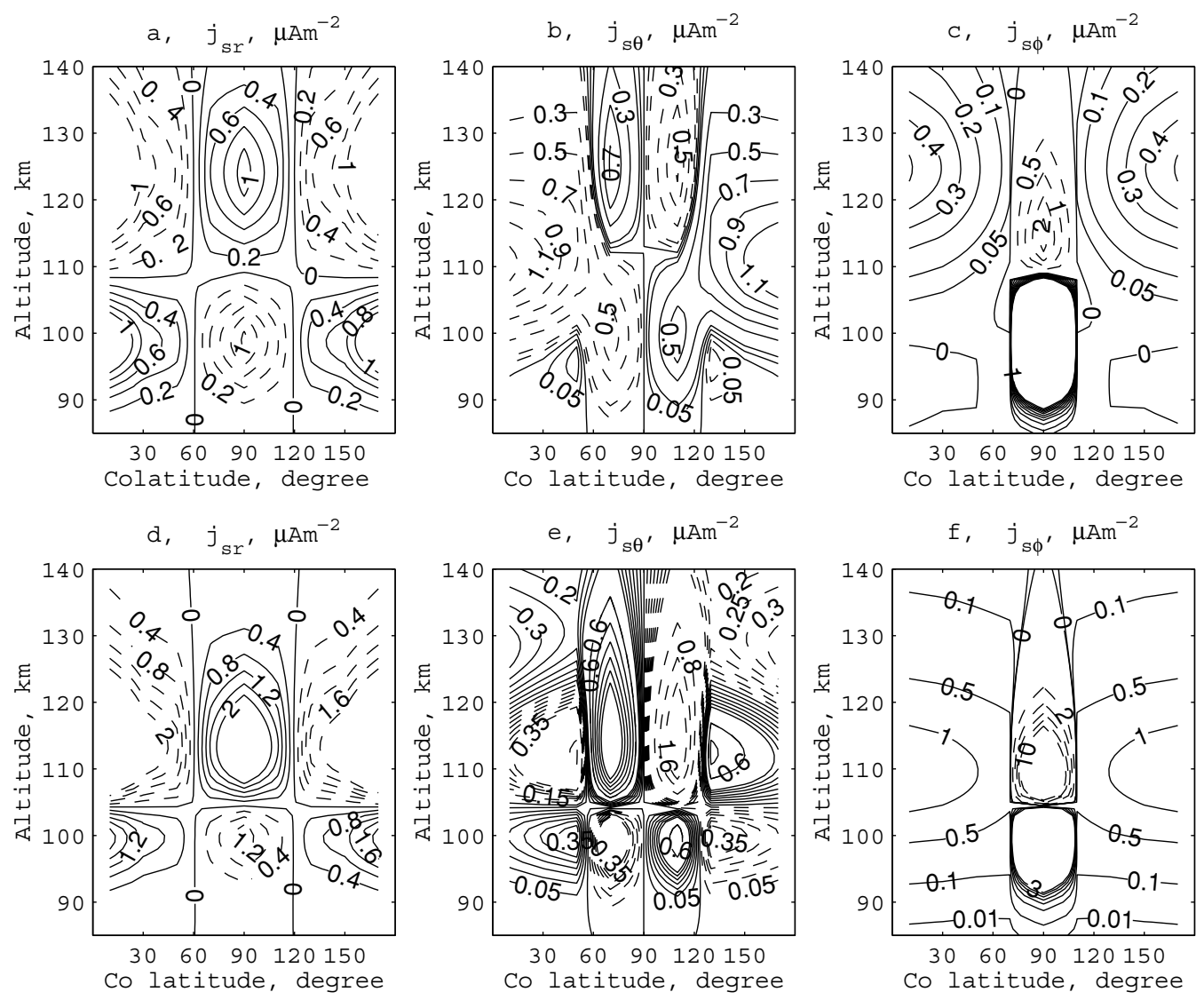

Fig. 3. As in Fig. 2 only in case of a potential electric field.

to expressions ( $R$ is radius of the Earth)

$$
\Phi^{(0)}=-\frac{\partial \Delta_{\Omega} E^{(0)}}{\partial r}, \quad \Phi^{(1)}=-\frac{r^{2}}{R^{2}} j_{r}
$$

is necessary for the maintaining accuracy of numerical computations with the greatly varied (on some orders) conductivity value and at the presence of the conductivity jumps along the radial coordinate. Such situation arises, in particular, in a task of a global electromagnetic induction. There is a conductivity jump on the Earth-atmosphere boundary. The conductivity value changes on some orders both in an atmosphere, and inside the Earth.

The lower boundary was usually established in the carried out series of computations inside the Earth on the depth of $1500 \mathrm{~km}$ with zero values of potentials $E^{(0)}$ and $E^{(1)}$, that is equivalent to occurrence on this depth of the perfectly conducting base. It is possible, when the electromagnetic field of diurnal harmonics does not penetrate into the large depths at an Earth's conductivity distribution used.

Assuming a decrease of the potential at large $r \rightarrow \infty$, zero values of potentials $E^{(0)}$ and $E^{(1)}$ also were set on the upper boundary in the ionosphere. Hence, the ionosphere from above also becomes a perfectly conducting layer. An altitude of the upper boundary varied in various variants of simulations from 200 up to $800 \mathrm{~km}$ above a surface of the Earth, while the solution at small altitudes less than 150$200 \mathrm{~km}$ ceased to depend on a situation in the upper part.

Here it was not supposed to receive a complete conformity of simulated fields with observable ones at the ex- pense of the complication of a model by the detailed data. It was supposed to find out effective high-altitude boundaries of the ionospheric dynamo on the basis of the specified assumptions. As the test of the model is necessary, both the qualitative conformity of the calculated ground geomagnetic effect to an experimental picture and also concurrence of fields under the order of observable values was checked.

With the purpose of a simplification of the result analysis, the field of winds was set at heights more than $80 \mathrm{~km}$ for the diurnal harmonic on the frequency $\omega$ by the following expressions:

$$
\begin{gathered}
u_{\theta}=130 \cos \theta \exp [i(\varphi+\omega t)], \\
u_{\varphi}=130 i \exp [i(\varphi+\omega t)], \quad m s^{-1},
\end{gathered}
$$

reflecting a main effect of a movement of the warm air into the night hemisphere. Here $t$ is Universal Time, so a local midday is at $180^{\circ} \mathrm{E}$ when $t=0$. External initial dynamosource currents are $\mathbf{J}=\mu \widehat{\sigma} \mathbf{u} \times \mathbf{H}_{E}$, where $\mathbf{u}$ is a wind vector and $\mathbf{H}_{E}$ is an Earth's magnetic field vector.

The used high-altitude profiles of the longitudinal $\sigma_{0}$, Pedersen $\sigma_{P}$ and Hall $\sigma_{H}$ conductivity (Fig. 1) corresponded to the typical average conditions in the ionosphere and did not depend on a longitude and latitude. The centered dipole model with combining geographical and geomagnetic poles was used for the geomagnetic field $\mathbf{H}_{E}$.

In an atmosphere, the conductivity model is $\sigma(h)=$ $\sigma_{0} \exp (h / H)$ in all simulations where $h$ is an altitude in $\mathrm{km}$ above the Earth's surface, $\sigma_{0}=10^{-14} \mathrm{~S} \mathrm{~m}^{-1}$ and $H=6$ 

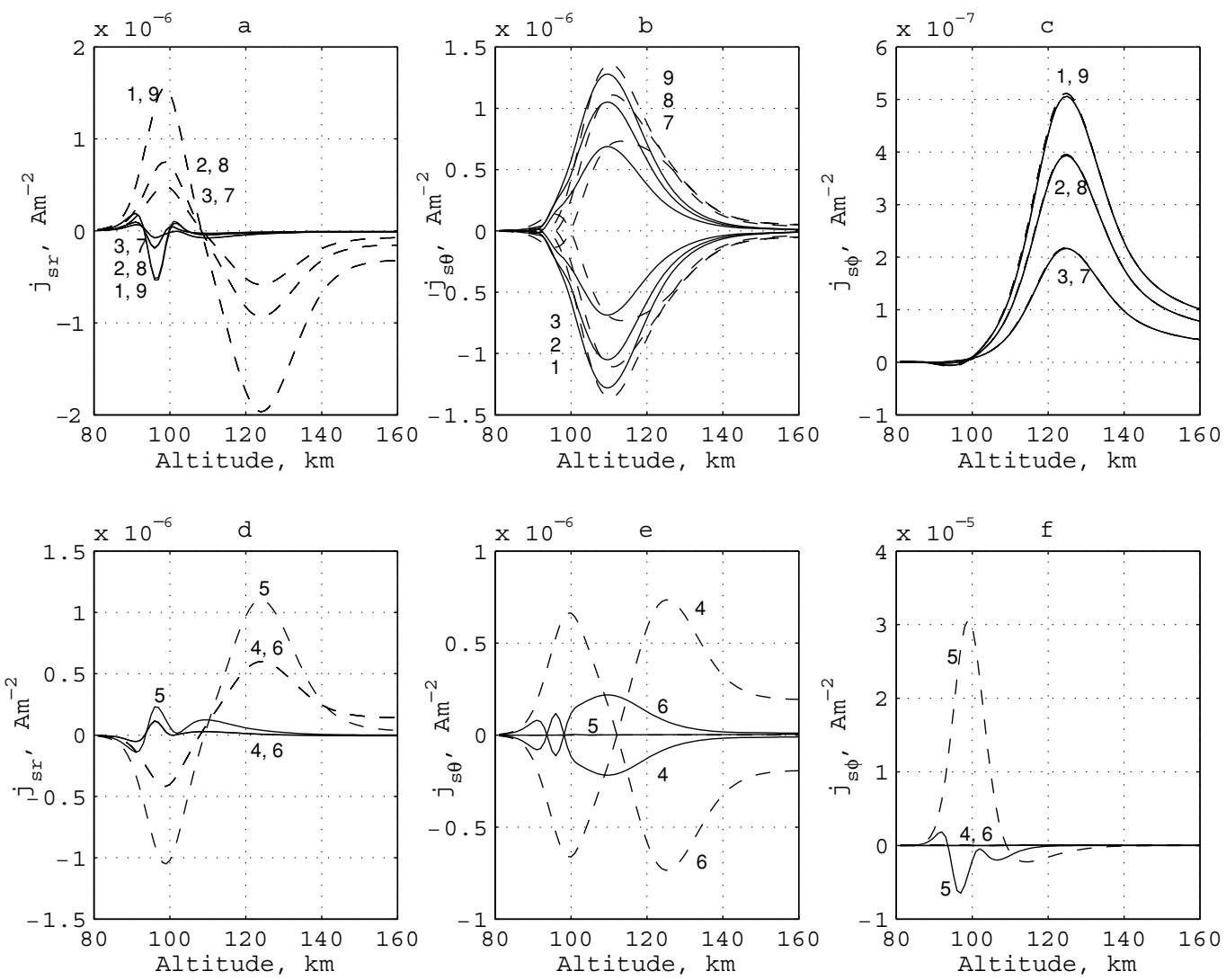

Fig. 4. Radial profiles of moduli of the $j_{s r}$ component (a and d), of the $j_{s \theta}$ component ( $\mathrm{b}$ and e) and of the $j_{s \varphi}$ component (c and f) of the total current of the ionospheric dynamo for longitude $0^{\circ}$ and several latitudes, which are specified by numbers correspond 1 to $80^{\circ} \mathrm{N}, 2$ to $60^{\circ} \mathrm{N}, 3$ to $40^{\circ} \mathrm{N}, 4$ to $20^{\circ} \mathrm{N}, 5$ to $0^{\circ}, 6$ to $20^{\circ} \mathrm{S}, 7$ to $40^{\circ} \mathrm{S}, 8$ to $60^{\circ} \mathrm{S}$, and 9 to $80^{\circ} \mathrm{S}$ (solid lines correspond to the account of a vortical electric field of the magnetic mode, dashed lines correspond to case of a potential electric field). Profiles (a, b and c) concern to latitudes large of 40 degrees and profiles (d, e and f) concern to low latitudes. Altitudes above the Earth's surface are indicated. The $j_{s r}$ components (a and d) and the $j_{s \varphi}$ components (c and f) coincide for symmetric northern and southern latitudes, and the $j_{s \theta}$ components ( $\mathrm{b}$ and e) change a sign on opposite in this situation.

$\mathrm{km}$. This model is matched with the appropriate profiles in the ionosphere using a spline interpolation. Both a model of a homogeneous sphere and the exponential Lahiri\&Price model $\sigma(h)=\sigma_{0} \exp (h /(R / 55))$ where $\sigma_{0}=10^{-3} S \mathrm{~m}^{-1}$ and $h$ is a depth in $\mathrm{km}$ (Rokityansky, 1982) was used as a spherical symmetric part of the conductivity deep distribution in the Earth. The calculations were carried out also at presence of the lateral heterogeneity of the conductivity of the Earth.

A grid size of 20 degrees in co-latitude and 30 degrees in longitude is used. For increase of the calculation accuracy it is possible to reduce a grid size on angles and radius, but it will require also large computational resources. The calculations with a step of 9 degrees in co-latitude required up to 12 hours on the computer with the $1200 \mathrm{MHz}$ processor. The calculation accuracy of $\sim 1 \%$ was achieved, when the radial step was of $0,1 \mathrm{~km}$ in an atmosphere, and of 3 $\mathrm{km}$ in the Earth. The reduction of time of the calculation is possible in the further by using splitting methods.

\section{Results of numerical simulations}

\subsection{About skin effect in the ionosphere}

An electromagnetic induction is usually considered at dynamo modelling only as a source of dynamo fields arising at movement of the conducting medium in the geomagnetic field. However temporal oscillations of a magnetic field of geomagnetic variations can generate according to the law of an electromagnetic induction also a vortical electric field and currents at the large conductivity of media. In particular, a skin effect of fields of geomagnetic variations is essential in the Earth. The similar effect is also essential in the ionosphere, if the skin depth is comparable to the height thickness of the layer, where ionospheric dynamo currents are concentrated. Therefore an electric field was not supposed potential in the anisotropic conducting ionosphere in the present paper.

The simulations have shown that the vortical electric field of the diurnal geomagnetic Sq-variation is compared with its potential electric field in upper layers of localization of ionospheric dynamo currents. Fig. 1 shows high-altitude profiles of conductivities used by simulations, and received high-altitude profiles of component moduli of the electric field of both modes for several latitudes. It is visible, that the skin effect plays an appreciable role in upper ionospheric layers for the radial component of the electric field specially.

Total current distributions are shown for all components in cases of the non-potential (Fig. 2) and potential (Fig. 3) electric field. The total current, concerning which equation (6) was solved, is $\mathbf{j}_{s}=\mathbf{j}+\mathbf{J}$, i.e. the sum of currents of electrical and magnetic modes and an initial external dynamosource current. The calculations for both cases were carried 

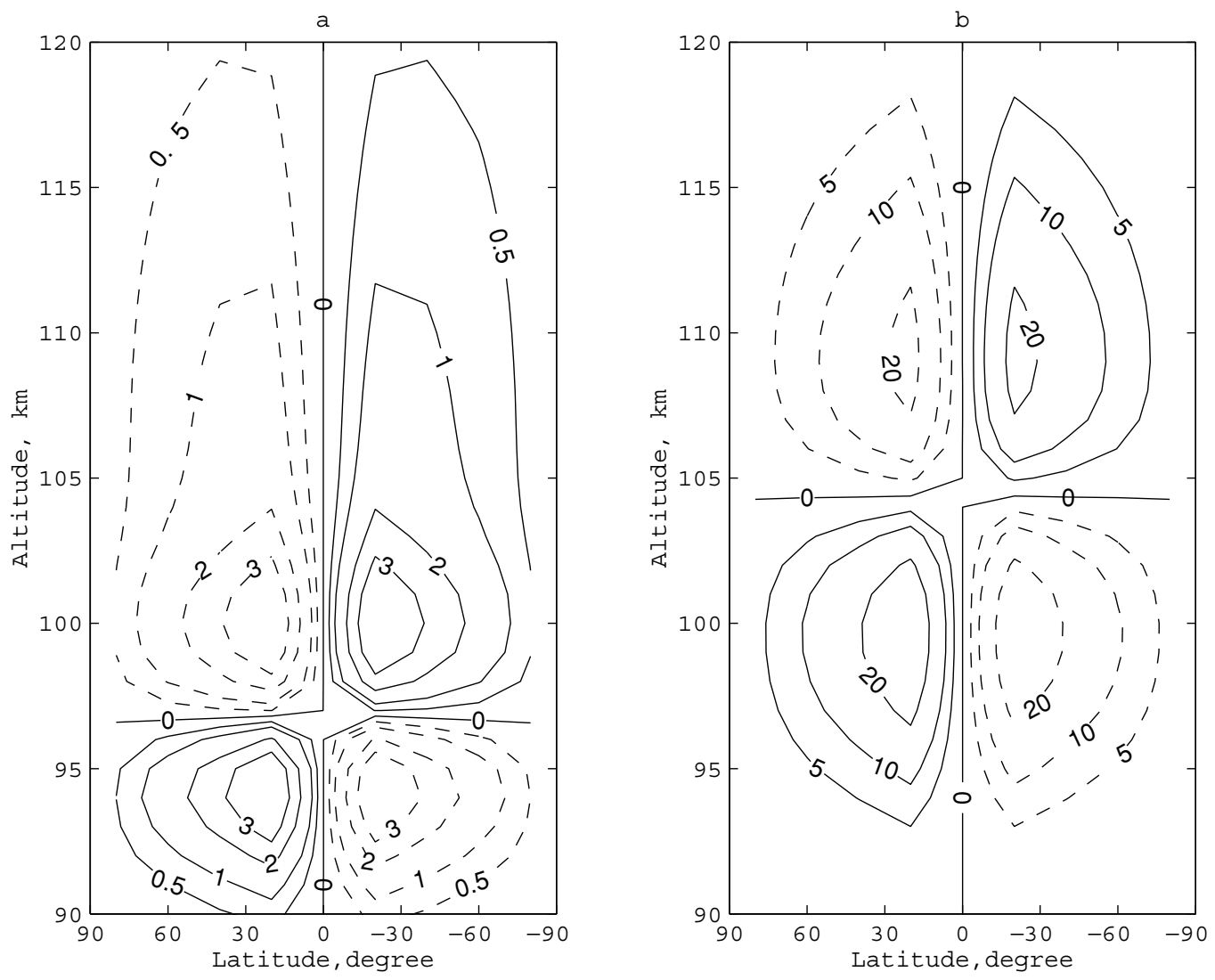

Fig. 5. Distributions of stream functions (in $\mathrm{A} \mathrm{m}^{-1}$ ) of toroidal horizontal currents in the ionosphere for morning (a and $\mathrm{b}$ ) and for evening (the same distributions with opposite signs of functions) in view of ionospheric skin effect (a) and without it (b).

out on the same numerical model, but the frequency was set equal $10^{-16} \mathrm{~Hz}$ for a potential electric field. Pictures are shown at night (top) and morning (bottom) meridians. Pictures at midday and evening meridians are similar, respectively, and differ only by change of signs at components due to the simplification and symmetry of the model. As initial dynamo-source currents are symmetric in northern and southern hemispheres, total current systems also have this symmetry. These pictures specify a three-dimensional character of the current system. Radial (high-altitude) currents are less than horizontal currents, and this ratio amplifies in case of a non-potential electric field. Horizontal currents represent several contrary vortices with different localization in height and latitude depending on longitude. Let us note the certain qualitative similarity of current systems for potential and non-potential cases. At the same time, current vortices are narrower on height in a non-potential case. Also it is observed smoother transitions between current vortices. Probably a skin effect on horizontal coordinates influences these circumstances.

It is necessary to emphasize, that the contribution of an electric field of the magnetic mode (of a vortical field) in a current system is absent in the potential case, and all system is created only by a field of the electrical mode. The distinctions of current systems in both cases are visible also from Fig. 4. Here some radial profiles of all components of the total current are shown by solid lines for a case including a vortical field and by dashed lines for a potential case. Radial currents are decreased for the non-potential electrical field. Also horizontal current components are decreased at low latitudes in this case.

\subsection{About ground-level geomagnetic effect}

Let us note, that the calculated current system $\mathbf{j}_{s}$ can be expanded by two current subsystems if take advantage of the formulas similar to relations (1)

$$
\begin{aligned}
& j_{s \theta}=\frac{1}{r} \frac{\partial J^{(1)}}{\partial \theta}+\frac{1}{r \sin \theta} \frac{\partial J^{(0)}}{\partial \varphi}, \\
& j_{s \varphi}=\frac{1}{r \sin \theta} \frac{\partial J^{(1)}}{\partial \varphi}-\frac{1}{r} \frac{\partial J^{(0)}}{\partial \theta} .
\end{aligned}
$$

One poloidal subsystem with irrotational horizontal currents with the potential function $J^{(1)}$ combined with a radial current forms a closed circuit. Other toroidal subsystem of divergence-free horizontal currents with the stream function $J^{(0)}$ is a complete closed current circuit. Taking into account, that

$$
\begin{gathered}
\left(\nabla \times \mathbf{j}_{s}\right)_{r}=-\frac{1}{r^{2}} \Delta_{\Omega} J^{(0)} \\
\nabla \cdot \mathbf{j}_{s}=\frac{1}{r^{2}} \frac{\partial}{\partial r}\left(r^{2} j_{s r}\right)+\frac{1}{r^{2}} \Delta_{\Omega} J^{(1)}=0 \\
\nabla \cdot \mathbf{H}=\frac{1}{r^{2}} \frac{\partial}{\partial r}\left(r^{2} H_{r}\right)+\frac{1}{r^{2}} \Delta_{\Omega} H^{(1)}=0, \\
(\nabla \times \nabla \times \mathbf{H})_{r}=\frac{1}{r^{2}} \Delta_{\Omega}\left(\frac{\partial H^{(1)}}{\partial r}-H_{r}\right),
\end{gathered}
$$



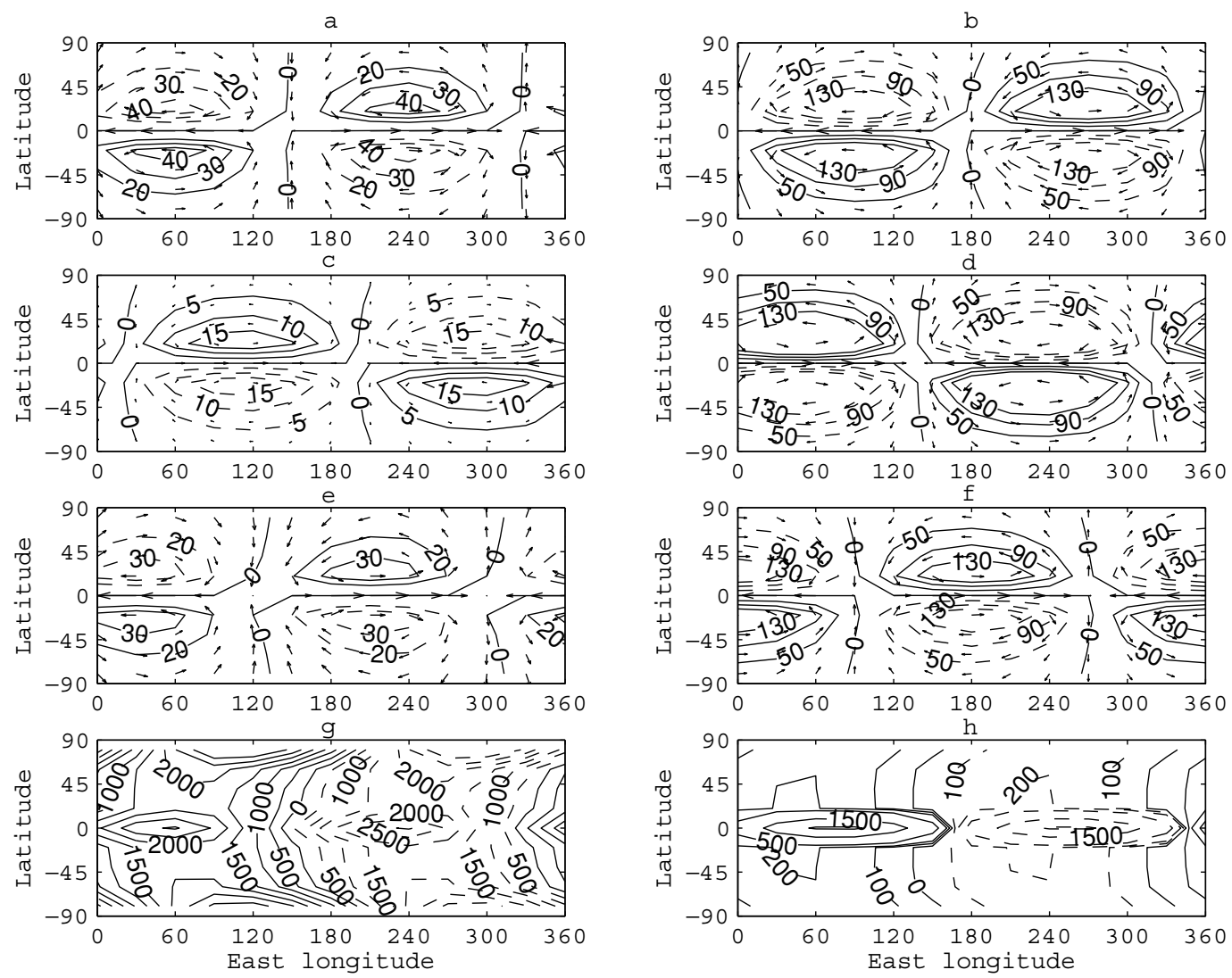

Fig. 6. Contours of height-integrated stream functions (in $k A$ ) of toroidal horizontal currents for upper (a and b), lower (c and d), and both totally (e and $\mathrm{f}$ ) vortices. Contours of the electric potential (in $V$ ) of the electrical mode ( $\mathrm{g}$ and $\mathrm{h}$ ). Contours are shown by dashed lines for negative values and by solid lines for positive values in view of ionospheric skin effect (a, c, e and g) and without it (b, d, f and h). The vectors in maps (a, b, c, d, e and f) represent toroidal horizontal currents. Potential distributions concern to heights of $145 \mathrm{~km}(\mathrm{~g})$ and $155 \mathrm{~km}(\mathrm{~h})$.

we shall receive the following equations for potentials of a magnetic field:

$$
\begin{gathered}
-\frac{1}{r^{2}} \Delta_{\Omega} H^{(0)}=j_{s r}, \quad \frac{\partial H^{(0)}}{\partial r}=J^{(1)} \\
\frac{1}{r^{2}} \frac{\partial^{2}}{\partial r^{2}}\left(r^{2} H_{r}\right)+\frac{1}{r^{2}} \Delta_{\Omega} H_{r}=\frac{1}{r^{2}} \Delta_{\Omega} J^{(0)} \\
\frac{1}{r^{2}} \frac{\partial}{\partial r}\left(r^{2} \frac{\partial H^{(1)}}{\partial r}\right)+\frac{1}{r^{2}} \Delta_{\Omega} H^{(1)}=-\frac{1}{r^{2}} \frac{\partial}{\partial r}\left(r^{2} J^{(0)}\right) .
\end{gathered}
$$

Equations (12) allow to find the magnetic field of the electrical mode, and equations (13) and (14) allow to find the magnetic field of the magnetic mode, if the spatial distribution of the total current $\mathbf{j}_{s}$ is calculated. Thus functions $J^{(0)}$ and $J^{(1)}$ in right-hand sides (12)-(14) can be received according to (10) from the equations:

$$
\begin{aligned}
& \frac{1}{r} \Delta_{\Omega} J^{(1)}=\frac{1}{\sin \theta}\left(\frac{\partial j_{s \varphi}}{\partial \varphi}+\frac{\partial}{\partial \theta}\left(\sin \theta j_{s \theta}\right)\right), \\
& \frac{1}{r} \Delta_{\Omega} J^{(0)}=\frac{1}{\sin \theta}\left(\frac{\partial j_{s \theta}}{\partial \varphi}-\frac{\partial}{\partial \theta}\left(\sin \theta j_{s \varphi}\right)\right) .
\end{aligned}
$$

From here it is visible, that the magnetic field of the electrical mode is determined by the poloidal current system, and the field of the magnetic mode is defined by toroidal current system. The equations (12)-(14) can be solved using a decomposition by spherical functions $Y_{n}(\Omega)$, where $\Omega$ are spherical angular coordinates. If with account of (11) to present calculated currents $\mathbf{j}_{s}$ as

$$
\begin{gathered}
J^{(0)}(r, \Omega)=\sum_{n=1}^{\infty} J_{n}^{(0)}(r) Y_{n}(\Omega), \\
J^{(1)}(r, \Omega)=\sum_{n=1}^{\infty} J_{n}^{(1)}(r) Y_{n}(\Omega), \\
j_{s r}(r, \Omega)=\sum_{n=1}^{\infty} Y_{n}(\Omega) \frac{n(n+1)}{r^{2}} \int_{0}^{r} J_{n}^{(1)}(x) d x,
\end{gathered}
$$

that the decompositions are fair for magnetic mode below then spherical current shell with boundary radiuses $r_{l}$ and $r_{u}$ and currents described by the first formula (15):

$$
\begin{gathered}
H^{(1)}(r, \Omega)=\sum_{n=1}^{\infty} Y_{n}(\Omega) \frac{n+1}{2 n+1} \int_{r_{l}}^{r_{u}}\left(\frac{r}{x}\right)^{n} J_{n}^{(0)}(x) d x . \\
H_{r}(r, \Omega)=\frac{1}{r} \sum_{n=1}^{\infty} Y_{n}(\Omega) \frac{n(n+1)}{2 n+1} \int_{r l}^{r u}\left(\frac{r}{x}\right)^{n} J_{n}^{(0)}(x) d x .
\end{gathered}
$$

The equations (12) have the solution for the electrical mode:

$$
H^{(0)}(r, \Omega)=\sum_{n=1}^{\infty} Y_{n}(\Omega) \int_{0}^{r} J_{n}^{(1)}(x) d x .
$$



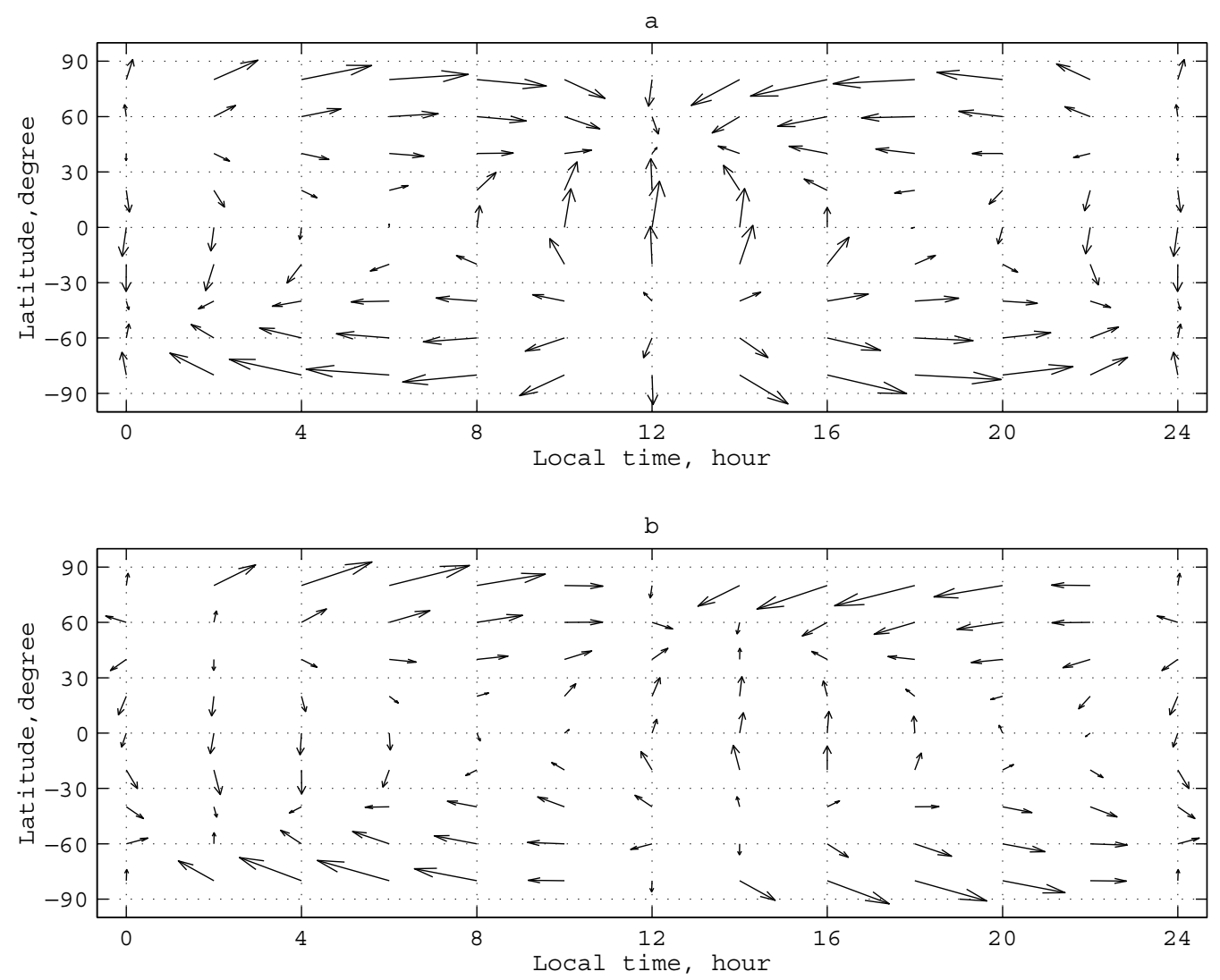

Fig. 7. Maps of calculated ground-level geomagnetic effect of diurnal variations in view of the vortical electric field in the ionosphere (a) and without it (b).

From comparison of last equation (15) and (17) it is visible, that the magnetic field of the electrical mode is determined by value of a radial current. It is usually considered, referring on (Fukushima, 1976), that under the ionosphere magnetic fields of currents, radial and closing them spreading on ionosphere, compensate each other, and consequently the poloidal current subsystem does not create a ground geomagnetic effect. As it is visible from the received solution (17), it will be correct at any current distributions in the ionosphere, if to neglect radial currents to the Earth through a poorly conducting atmosphere and magnetic fields, created by them. It is known also (Parkinson, 1983), that the toroidal magnetic field should not leave conducting medium namely the ionosphere in this case. Taking into account the first equation of system (11), we conclude, that in this approach the ground geomagnetic effect (16) is really caused only by vortical part of total current system, that is, by the toroidal current subsystem. Therefore current systems with an identical toroidal part will be equivalent, as create the same magnetic field on a terrestrial surface.

Let us compare toroidal current subsystems for cases in view of vortical electric fields and without it. Distributions of stream functions $J^{(0)}$ of toroidal horizontal currents in the ionosphere are shown for morning-evening meridians in view of the vortical electric field (Fig. 5a) and without such account (Fig. 5b). Qualitatively these distributions coincide, forming a system from upper and lower contrary vortices. But they differ both on amplitude of vortices, and on height (of 97 and $104 \mathrm{~km}$ respectively), dividing these contrary vortices. Maps of height-integrated stream function of toroidal horizontal currents are shown in Fig. 6 for upper vortex (a and b), for lower vortex (c and d), and for both vortices (e and f) totally. Also maps of electric potential of the electrical mode are shown ( $g$ and $h)$. All maps are shown in view of skin effect in the ionosphere (a, c, e and g) and without it (b, d, f and h). Potential distributions ( $g$ and $h$ ) are similar in a measure (here were taken into account only first diurnal harmonic) to known empirical maps of ionospheric potential by (Richmond et al., 1980).

Maps of calculated ground geomagnetic effect coincide qualitatively with each other and with a picture, observable in practice (Parkinson, 1983). They are shown in Fig. 7 for non-potential (a) and for potential (b) cases. Magnetic field components are calculated here using the first equation (4) and two last equations (1). In the middle and low latitudinal regions, it is possible to observe that strength of magnetic field vectors is large as though in case of non-potential electric field compared with that of potential one. This result does not contradict the conventional influence of the inductive electric fields. It is possible to conclude from Fig. 7 that apparently the induction fields in the Earth do not influence the removed dynamo source in the ionosphere. In particular, it is known that the contribution of the induction fields in the Earth in ground-level geomagnetic effect makes no more than $30 \%$. Therefore the ground-level geomagnetic effect depends in the most part on properties of the ionospheric source. The induction fields try to reduce 

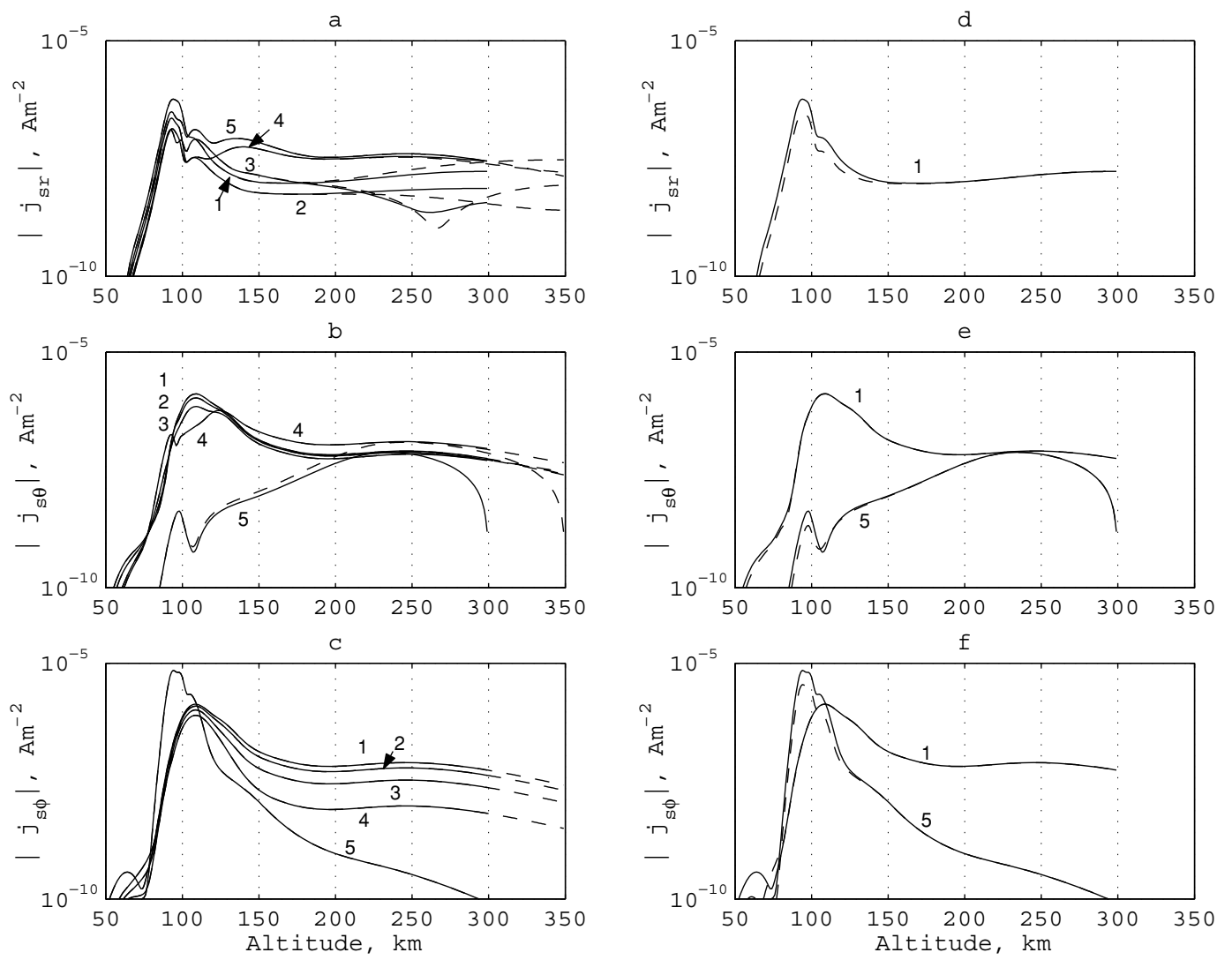

Fig. 8. Radial profiles of moduli of various components of the total current of the ionospheric dynamo received for two altitudes of the upper boundary condition (UBC) and two values of the radial step for longitude $0^{\circ}$ and several latitudes, which are specified by numbers correspond 1 to $80^{\circ} \mathrm{N}, 2$ to $60^{\circ} \mathrm{N}, 3$ to $40^{\circ} \mathrm{N}, 4$ to $20^{\circ} \mathrm{N}$ and 5 to $0^{\circ}$. The radial distances above the Earth's surface are indicated. The profiles (a, b and c) are shown by solid lines for the UBC altitude of $300 \mathrm{~km}$ and by dashed lines for the UBC altitude of $350 \mathrm{~km}$. They are calculated with a radial step of 100 meters. Profiles (d, e and f) are shown by solid lines for the radial step of 100 meters and by dashed lines for radial step of 50 meters.

this source as a whole. It is visible from the comparison of Fig. 6e and 6f. The maximal horizontal magnetic field on a surface of the Earth was about $10 \mathrm{nT}$ in both cases, but this value is less actually in the non-potential case.

\subsection{About effect of field-aligned currents between hemispheres}

Integration over a spherical surface of the first equation of the system (3) gives zero value of the total radial current through this sphere. At numerical approximation of the differential equations, it is necessary to prefer the finite differences automatically providing this condition. As it is not always possible, at numerical calculations it is necessary to supervise the total radial current through a spherical surface. The numerical value of radial current integrated over the round solid angle on any sphere did not exceed of $4 \pi 10^{-15} \mathrm{~A} \mathrm{~m}^{-2}$ in the carried out calculations.

At the same time, greatest ionospheric currents are of $\sim 10^{-6} \mathrm{~A} \mathrm{~m}^{-2}$ as was above shown. They are concentrated only in the ionosphere dynamo region on heights less than $150 \mathrm{~km}$, where the values of Hall and Pedersen conductivities are maximal at the given model. Amplitudes of currents decrease essentially at distance on a radial coordinate from the dynamo region both upward, and downward (Fig. 4). Thus it appears that the spatial distribution of ionospheric currents in the dynamo region practically does not depend on their behaviour in upper ionospheric layers with the large longitudinal conductivity. The change of the altitude of the upper boundary conditions of a task and also conductivity profiles or height decrease laws of Hall and Pedersen conductivities practically has not an effect on ionospheric currents in the dynamo region, though it is essential for a picture in upper layers. It is shown in Fig. 8, where the profiles are displayed describing accuracy and stability of calculations depending on the integration step and the altitude of the upper boundary condition. Therefore Sq-currents basically are located in the dynamo region of the ionosphere. Only small part of radial currents acts in higher layers. Let us note that the zero balance of radial currents through the removed spheres is provided at the expense of their compensation on different longitudes separately in northern and southern hemispheres. Thus current systems in regions above the altitude of the upper boundary condition are not taken into account here. In our opinion, it is insufficiently clear for the present, how these current systems can be constructed. But, as it is visible from calculations, the current densities in the basic dynamo region do not depend on the upper current systems. Therefore it is possible to approve, that the account of field-aligned currents and galvanic connections between the conjugated points in northern and southern hemispheres appears not essential to spatial distribution of ionospheric currents in the basic dynamo region. As the proof in Fig. 9 the same maps are shown, as in Fig. 2, but the results correspond to a case, when the conductivity values in the ionosphere were 

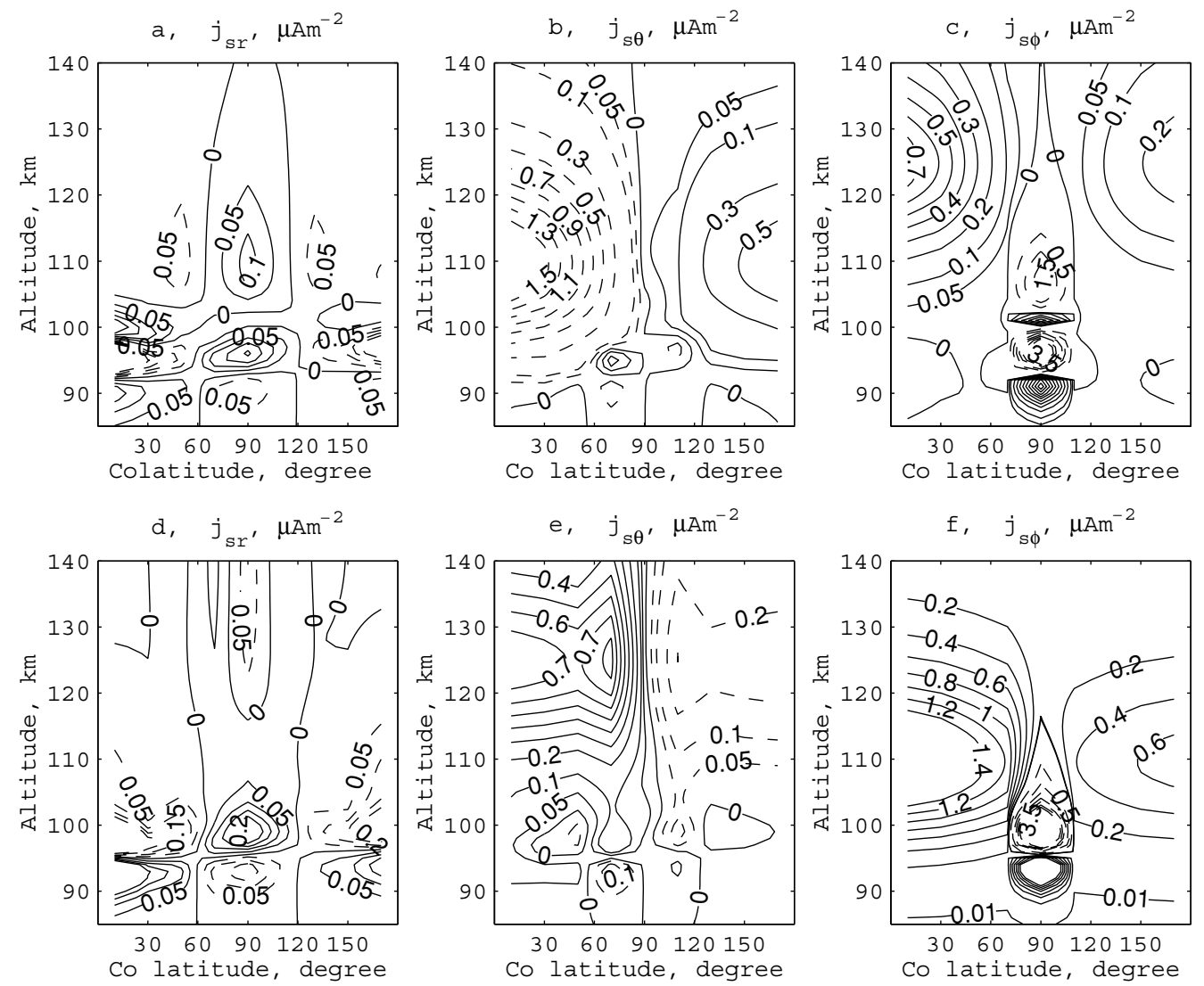

Fig. 9. As in Fig. 2, but the results correspond to a case, when the conductivity values in the ionosphere were increased by $50 \%$ in northern and reduced by $50 \%$ in southern hemisphere.

increased by $50 \%$ in northern and reduced by $50 \%$ in southern hemisphere.

Let us note at the same time, that the determination of currents in upper ionospheric layers causes difficulties. So, by Richmond and Roble (1987) it is marked that the map of field-aligned currents at height of $300 \mathrm{~km}$ received on their model differs from field-aligned currents found by Stening (1977) and Takeda (1982), which not coincide with each other. It is underlined, that the computation of field-aligned currents is quite sensitive to details of the wind and conductivity in the ionosphere, and also to the numerical technique used. A 2-D equation for potential received by integration of the initial equation along equipotential geomagnetic lines was solved by Takeda and Maeda (1980) and Takeda (1982) for the calculation of field-aligned currents. However at the altitudes of dynamo region of about $100 \mathrm{~km}$ and less, where the values of tensor conductivity components can be of one order, the field-aligned currents are comparable with the Hall and Pedersen currents. The condition of equipotential geomagnetic lines thus is broken, and the integration along these lines of coefficients of the initial equation for potential is incorrect. Therefore the three-dimensional current system in the ionosphere also were calculated in the present paper without using an approximation of equipotential geomagnetic lines.

\section{Conclusions}

It is shown that the two-mode electromagnetic numerical model taking into account coupled electrical and mag- netic modes in the anisotropic heterogeneous plasma is applicable for the solution of problem of the 3-D ionospheric dynamo without using the approximation of equipotential geomagnetic lines. This numerical model used the matrix flow run technique along the radial coordinate.

It is shown that the electromagnetic induction is not only source of dynamo effect, but also a reason of a non-potential electric field of Sq-variations and a skin effect in the ionosphere. Radial components of electric field are decreased due to a skin effect. Also horizontal currents are decreased at low latitudes in this case.

It is established that the toroidal current subsystem is responsible for ground-level geomagnetic effect of the ionospheric dynamo. The model gives pictures of ground-level geomagnetic effect which are qualitatively similar to observable ones.

It is shown that a three-dimensional current system generated by the dynamo action of winds is located basically at altitudes less than about $200 \mathrm{~km}$. It does not depend practically on an ionosphere condition in upper layers and weaker field-aligned currents between the northern and southern hemispheres. Hence an almost complete closed current circuit is generated in the dynamo layer.

Acknowledgments. This work was supported financially by RFBR grant N 04-05-64074. I thank an anonymous reviewer for useful comments on the manuscript. 


\section{References}

Fukushima, N., Generalized theorem for no ground magnetic effect of vertical currents connected with Pedersen currents in the uniform conducting ionosphere, Rep. Ionos. Space Res. Japan, 30, 35-40, 1976.

Parkinson, W. D., Introduction to Geomagnetism, Scottish Academic Press, Edinburg \& London, 1983.

Plotkin, V. V., Earth charge produced by thunderstorms, Russian Geology and Geophysics, 44, 257-264, 2003.

Richmond, A. D., M. Blanc, B. A. Emery, R. H. Wand, B. G. Fejer, R. F. Woodman, S. Ganguly, P. Amayenc, R. A. Behnke, C. Calderon, and J. V. Evans, An empirical model of quiet-day ionospheric electric fields at middle and low altitudes, J. Geophys. Res., 85, 4658-4664, 1980.

Richmond, A. D., E. C. Ridley, and R. G. Roble, A thermosphere/ionosphere general circulation model with coupled electrodynamics, Geophys. Res. Lett., 19, 601-604, 1992.

Richmond, A. D. and R. G. Roble, Electrodynamic effects of thermospheric winds from the NCAR Thermospheric General Circulation
Model, J. Geophys. Res., 92, 12365-12376, 1987.

Rokityansky I. I., Geoelectromagnetic Investigations of the Earth's Crust and Mantle, Springer-Verlag, Berlin, 1982.

Singh, A. and K. D. Cole, A numerical model of the ionospheric dynamo, J. Atmos. Terr. Phys., 49, 521-547, 1987.

Stening, R. J., Field-aligned currents driven by the ionospheric dynamo, $J$. Atmos. Terr. Phys., 39, 933-937, 1977.

Takeda, M., Three-dimensional ionospheric currents and field aligned currents generated by asymmetrical dynamo action in the ionosphere, $J$. Atmos. Terr. Phys., 44, 187-193, 1982.

Takeda, M. and M. Maeda, Three-dimensional structure of ionospheric currents. 1. Currents caused by diurnal tidal winds, J. Geophys. Res., 85, 6895-6899, 1980 .

V. Plotkin (e-mail: plotkin@uiggm.nsc.ru) 